\title{
Introgression of blast resistance genes into the elite rice variety MR263 through marker-assisted backcrossing
}

\begin{abstract}
BACKGROUND: Blast caused by the fungus Magnaporthe oryzae is a significant disease threat to rice across the world and is especially prevalent in Malaysia. An elite, earlymaturing, high-yielding Malaysian rice variety, MR263, is susceptible to blast and was used as the recurrent parent in this study. To improve MR263 disease resistance, the Pongsu Seribu 1 rice variety was used as donor of the blast resistance Pi-7(t), Pi-d(t)1 and Pir2-3(t) genes and qLN2 quantitative trait locus (QTL). The objective was to introgress these blast resistance genes into the background of MR263 using marker-assisted backcrossing with both foreground and background selection. RESULTS: Improved MR263-BR-3-2, MR263-BR-43, MR263-BR-13-1 and MR263-BR-26-4 lines carrying the Pi-7(t), Pi-d(t)1 and Pir2-3(t) genes and qLN2 QTL were developed using the simple sequence repeat (SSR) markers RM5961 and RM263 (linked to the blast resistance genes and QTL) for foreground selection and a collection of 65 polymorphic SSR markers for background selection in backcrossed and selfed generations. A background analysis revealed that the highest rate of recurrent parent genome recovery was $96.1 \%$ in MR263-BR-4-3 and 94.3\% in MR263-BR-3-2. CONCLUSION: The addition of blast resistance genes can be used to improve several Malaysian rice varieties to combat this major disease.
\end{abstract}

Keyword: Blast resistance; Molecular markers; Pi gene; Rice 